\title{
Early-onset severe hereditary sensory and autonomic neuropathy type 1 with S331F SPTLC1 mutation
}

\author{
BUM CHUN SUH ${ }^{1}$, YOUNG BIN HONG ${ }^{2}$, KHRIEZHANUO NAKHRO ${ }^{3}$, SOO HYUN NAM ${ }^{3}$, \\ KI WHA CHUNG ${ }^{3}$ and BYUNG-OK CHOI ${ }^{2}$ \\ ${ }^{1}$ Department of Neurology, Kangbuk Samsung Hospital, Sungkyunkwan University School of Medicine, Seoul 110-746;
${ }^{2}$ Department of Neurology, Samsung Medical Center, Sungkyunkwan University School of Medicine, Seoul 135-710;
${ }^{3}$ Department of Biological Science, Kongju National University, Gongju, Chungnam 314-701, Republic of Korea
}

Received September 9, 2013; Accepted November 8, 2013

DOI: $10.3892 / \mathrm{mmr} .2013 .1808$

\begin{abstract}
Hereditary sensory and autonomic neuropathy type I (HSAN I) is an autosomal dominant disease characterized by prominent sensory impairment, resulting in foot ulcers or amputations and has a juvenile to adult onset. The major underlying causes of HSAN I are mutations in SPTLC1, which encodes the first subunit of serine palmitoyltransferase (SPT). To date, there have been no reports with regard to an HSAN patient of Korean origin. In this report we discussed an HSAN I patient with a missense mutation in SPTLC1 (c.992C>T: p.S331F). The patient had noticed frequent falls, lower leg weakness and hand tremors at age five. The patient also presented with foot ulcers, muscle hypotrophy, cataracts, hoarseness, vocal cord palsy and respiratory difficulties and succumbed to the condition at the age of 28 years. In accordance with previous reports, a mutation in Ser331 in the present patient was associated with early-onset and a severe phenotype. Therefore, Ser331 in SPTLC1 is a crucial amino acid, which characterizes the HSAN I phenotype.
\end{abstract}

\section{Introduction}

Hereditary sensory and autonomic neuropathy (HSAN), also known as hereditary sensory neuropathy, is a rare heterogeneous group of disorders with a wide range of clinical and genetic diversity (1). HSAN is traditionally classified into five subtypes (HSAN I-V) based on the age of the patient at onset, the pattern of inheritance and additional features

Correspondence to: Professor Byung-Ok Choi, Department of Neurology, Samsung Medical Center, Sungkyunkwan University School of Medicine, 50 Ilwon-dong Gangnam-Gu, Seoul 135-710, Republic of Korea

E-mail: bochoi@skku.edu

Professor Ki Wha Chung, Department of Biological Science, Kongju National University, 182 Sinkwan-dong, Gongju, Chungnam 314-701, Republic of Korea

E-mail: kwchung@kongju.ac.kr

Key words: hereditary sensory and autonomic neuropathy, SPTLC1, phenotype, mutation such as degree of motor and sensory impairment, autonomic dysfunction, particularly anhidrosis, and mental retardation (2). HSAN type I (HSAN I; MIM: 162400) is the most frequent HSAN subtype with autosomal dominant inheritance characterized by marked distal sensory impairment leading to painless ulceration, soft tissue infection and osteomyelitis in the hands and feet (3). As the disease progresses, a variable degree of distal muscle weakness and wasting with lancinating pain may be observed.

Mutations in SPTLC1 (MIM: 605712), which encodes the long-chain base subunit 1 of serine palmitoyltransferase (SPT), are the major underlying causes of HSAN I (1), and several mutations have been reported to be relevant in HSAN I: p.C133W, p.C133Y, p.C133R, p.V144D, p.S331F, pA310G, p.S331Y and p.A352V (2-13). SPT catalyzes serine and palmitoyl coenzyme A (CoA), which is the initial and rate-limiting step in the de novo biosynthesis of sphingolipids. The age of onset for HSAN I is usually in the second to fourth decades of life and motor involvement is variable and limited to the distal limbs. In contrast to typical HSAN I, three European cases of Ser331 mutations in SPTLC1 (two with p.S331F and one with p.S331Y) demonstrated an early onset and a severe phenotype (2,4-6).

In this study we report on the first known Korean HSAN I patient to harbor a p.S331F mutation in SPTLC1, with early onset and a severe phenotype.

\section{Patients and methods}

Patients. A Korean family with one HSAN I patient and three healthy individuals (ID: FC142, Fig. 1A) were enrolled in this study. Healthy Korean controls with no familial history of neuromuscular disorders $(n=300)$ were also recruited for this study. Paternity was confirmed by the genotyping of 15 microsatellites using a PowerPlex 16 system (Promega, Madison, WI, USA). Written informed consent was obtained from all participants according to the protocol approved by the Institutional Review Board for Ewha Womans University, Mokdong Hospital (ECT 11-58-37; B.O. Choi).

Clinical and electrophysiological assessments. The patient and the patient's sister and parents were examined for motor 
A

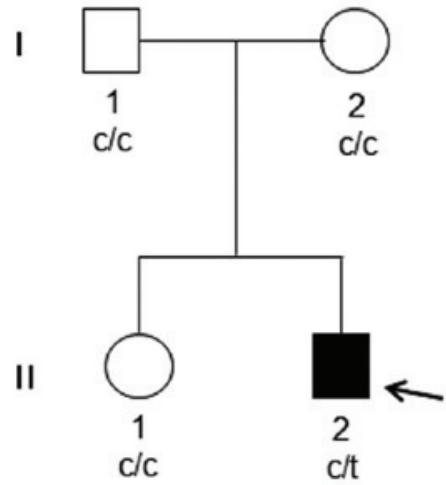

Como sapiens (NP 006406.1)
Pongo abeli (NP 001126393.1)
Cricetulus griseus (NP 001233688.1)
Mus musculus (NP 033295.2)
Rattus norvegicus (NP 001101876.1)
Bos taurus (NP 001029921.1)
$\quad$ Gallus gallus (XP 423815.3)
$\quad$ Canis lupus familiaris (XP 541308.2)
$\quad$ Xenopus laevis (NP 001084963.1)
Danio rerio (NP 001018307.1)
Drosophila melanogaster (NP 610842.1)
Caenorhabditis elegans (NP 001021978.1)
B

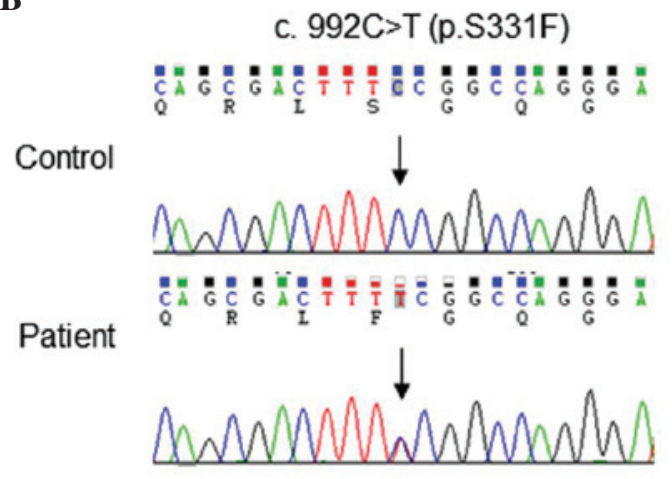

RSF V I DHQR LSGQGYCFSASL RSF V I DHQR L SGQGYCFSAS L RSFVVDHQRLSGQGYCFSASL RSFVVDHQRLSGQGYCFSASL RSFVVDHQRLSGQGYCFSASL RSFV I DHQR LSGQGYCFSAS L RSF I I DHQRLSGQGYCFSAS L RSFV I DHQRLSGQGYCFSAS L RSFVIDHQR LSGQGYCFSAS L RSFVIDHQRLSGQGYCFSAS L SHF I AEHQRLSGLGY I FSAS L RS Y V VGHQR LSGLGYCFSAS L

Figure 1. Pedigree, sequencing chromatogram of the SPTLC1 gene mutation in the FC142 family. (A) The pedigree of the FC142 family. The open symbols represent unaffected individuals while the filled symbols represent affected individuals. The proband is indicated by an arrow. The genotypes of the SPTLC1 mutation are indicated below each examined member. (B) Sequencing chromatograms. Vertical arrows indicate the mutation site. (C) Conservation analysis of the amino acid sequences between different species. The analysis was conducted using MEGA5 version 5.05. The mutation site and its neighboring sequences were well conserved among the different species.

and sensory impairments, deep tendon reflex abnormalities and muscle atrophy. The strength of the flexor and extensor muscles was assessed manually using the Medical Research Council scale (14). Physical disabilities were assessed using the functional disability scale (FDS) (15). Nerve conduction studies (NCS) were performed on all family members of FC142 with a surface electrode (Ambu, Ballerup, Denmark). Motor nerve conduction velocities (MNCVs) of the median and ulnar nerves were determined by providing stimulation at the elbow and wrist while recording the compound muscle action potentials (CMAPs) over the abductor pollicis brevis and adductor digiti quinti, respectively. In the same manner, the MNCVs and CMAPs of the fibular and tibial nerves were determined. Sensory nerve conduction velocities and sensory nerve action potentials (SNAPs) of the median, ulnar and sural nerves were obtained.

DNA preparation and pre-screening for Charcot-Marie-Tooth (CMT) disease genes. DNA was purified from blood samples using a QIAamp blood DNA purification kit (Qiagen, Hilden, Germany). The patient's DNA was pre-screened for a $1.4 \mathrm{Mbp}$ length of $17 \mathrm{p} 12$ duplication/deletion, a major genetic cause of CMT, by using hexaplex microsatellite polymerase chain reaction (16).

Exome sequencing and identification of causative mutation. Exome sequencing was performed in the male patient (II-2) according to the previous report (17). Exome capturing was achieved using a SeqCap EZ, version 3.0 (Roche-NimbleGen,
Table I. Summary of exome sequencing for the HSAN I patient (II-2).

Items II-2

Total yields $6.14 \mathrm{Gbp}$

Mappable reads $93.1 \%$

Target coverage $(\geq 10 \mathrm{x})$ $94.3 \%$

Total observed SNP number 57705

Coding SNP number 19757

Total observed indel number 10450

Coding indel number 588

Functionally significant variants in CMT genes ${ }^{\mathrm{a}}$ 32

${ }^{\mathrm{a}}$ Observed number of functionally significant variants (missense, nonsense, coding indels, and splicing site) in >60 CMT related genes. HSAN, hereditary sensory and autonomic neuropathy; SNP, single nucleotide polymorphisms; CMT, Charcot-Marie-Tooth disease.

Madison, WI, USA), and next generation sequencing was performed using a HiSeq 2000 Genome analyzer (Illumina, SanDiego,CA,USA).The UCSCassemblyhg19(NCBIbuild 37.1) was used as the reference sequence with BWA aligner software (http://bio-bwa.sourceforge.net/). Variant calling was achieved using a SAMtools program (http://samtools.sourceforge.net/). Single nucleotide polymorphisms (SNPs) with a quality value $>20$ were considered as candidates. 
Table II. Functionally significant variants observed in CMT genes for the FC142 family (II-2).

\begin{tabular}{|c|c|c|c|c|c|c|c|}
\hline \multirow[b]{2}{*}{ Gene } & \multirow[b]{2}{*}{ RefSeq $^{a}$} & \multirow[b]{2}{*}{ Chr: position } & \multicolumn{2}{|l|}{ Variants } & \multirow[b]{2}{*}{ dbSNP137 } & \multirow[b]{2}{*}{$1000 \mathrm{G}^{\mathrm{c}}$} & \multirow[b]{2}{*}{ Description } \\
\hline & & & $\mathrm{Nt}^{\mathrm{b}}$ & $\mathrm{AA}$ & & & \\
\hline$N G F$ & NM_002506.2 & chr01:115829313 & c. $104 \mathrm{C}>\mathrm{T}$ & $\mathrm{A} 35 \mathrm{~V}$ & rs6330 & 0.31 & Polymorphism \\
\hline OPA1 & NM_130834.2 & chr03:193334991 & c. $473 \mathrm{G}>\mathrm{A}$ & $\mathrm{S} 158 \mathrm{~N}$ & rs7624750 & 0.46 & Polymorphism \\
\hline FIG4 & NM_014845.5 & chr06:110064928 & c. $1090 \mathrm{~A}>\mathrm{T}$ & M364L & rs2295837 & 0.10 & Polymorphism \\
\hline FIG4 & - & chr06:110107517 & c. $1961 \mathrm{~T}>\mathrm{C}$ & V654A & rs9885672 & 0.37 & Polymorphism \\
\hline$N E F L$ & NM_006158.4 & chr08:24811071 & c.1407_1408insC & P471fs & rs 11300136 & - & Polymorphism \\
\hline SPTLC1 & NM_006415.2 & chr09:94809543 & c. $992 \mathrm{C}>\mathrm{T}$ & $\mathrm{S} 331 \mathrm{~F}$ & - & - & Causative \\
\hline$I K B K A P$ & NM_003640.3 & chr09:111659439 & c. $2490 A>G$ & I830M & rs2230794 & 0.08 & Polymorphism \\
\hline$I K B K A P$ & - & chr09:111659483 & c. $2446 \mathrm{~A}>\mathrm{C}$ & I816L & rs2230793 & 0.29 & Polymorphism \\
\hline$I K B K A P$ & - & chr09:111660851 & c. $2294 \mathrm{G}>\mathrm{A}$ & $\mathrm{G} 765 \mathrm{E}$ & rs2230792 & 0.28 & Polymorphism \\
\hline$I K B K A P$ & - & chr09:111665169 & c. $1804 \mathrm{C}>\mathrm{T}$ & R602W & rs200397694 & 0.00 & Non-cosegregation \\
\hline LRSAMI & NM_138361.5 & chr09:130242166 & c. $952 \mathrm{~A}>\mathrm{G}$ & N318D & rs 1539567 & 0.74 & Polymorphism \\
\hline SETX & NM_015046.5 & chr09:135139901 & c. $7759 A>G$ & I2587V & rs 1056899 & 0.51 & Polymorphism \\
\hline SETX & - & chr09:135173685 & c. $5563 \mathrm{~A}>\mathrm{G}$ & $\mathrm{T} 1855 \mathrm{~A}$ & rs2296871 & 0.41 & Polymorphism \\
\hline SETX & - & chr09:135203530 & c. $3455 \mathrm{~T}>\mathrm{G}$ & $\mathrm{F} 1152 \mathrm{C}$ & rs3739922 & 0.10 & Polymorphism \\
\hline SETX & - & chr09:135205006 & c. $1979 \mathrm{C}>\mathrm{G}$ & A660G & rs882709 & 0.21 & Polymorphism \\
\hline DHTKDI & NM_018706.6 & chr10:12111090 & c. $.58 \mathrm{~T}>\mathrm{C}$ & F20L & rs 1279138 & 0.98 & Polymorphism \\
\hline DHTKDI & - & chr10:12131081 & c. $814 \mathrm{~T}>\mathrm{G}$ & Y272D & rs3740015 & 0.47 & Polymorphism \\
\hline DHTKDI & - & chr10:12143105 & c. $1821 \mathrm{C}>\mathrm{G}$ & I607M & rs2062988 & 0.72 & Polymorphism \\
\hline$S B F 2$ & NM_030962.3 & chr11:9853777 & c. $3646 \mathrm{C}>\mathrm{G}$ & Q1216E & rs 12574508 & 0.10 & Polymorphism \\
\hline$I G H M B P 2$ & NM_002180.2 & chr11:68678962 & c. $602 \mathrm{~T}>\mathrm{C}$ & L201S & rs560096 & 0.70 & Polymorphism \\
\hline$I G H M B P 2$ & - & chr11:68705674 & c. $2636 \mathrm{C}>\mathrm{A}$ & T879K & rs 17612126 & 0.23 & Polymorphism \\
\hline$W N K 1$ & NM_213655.4 & chr12:990912 & c. $3922 \mathrm{~A}>\mathrm{C}$ & $\mathrm{T} 1308 \mathrm{P}$ & rs956868 & 0.85 & Polymorphism \\
\hline WNK1 & - & chr12:994487 & c. $5273 \mathrm{G}>\mathrm{C}$ & C1758S & rs7955371 & 0.99 & Polymorphism \\
\hline$K A R S$ & NM_005548.2 & chr16:75669878 & c. $601 \mathrm{~T}>\mathrm{C}$ & Y201H & rs 150529876 & 0.00 & Polymorphism \\
\hline SEPT9 & NM_001113495.1 & chr17:75494705 & c. $1390 A>G$ & M464V & rs 2627223 & 0.92 & Polymorphism \\
\hline$C T D P 1$ & NM_048368.3 & chr18:77473127 & c. $1019 \mathrm{C}>\mathrm{T}$ & T340M & rs2279103 & 0.11 & Polymorphism \\
\hline$C T D P 1$ & - & chr18:77474626 & c. $1166 \mathrm{C}>\mathrm{T}$ & A389V & rs 144647072 & 0.02 & Polymorphism \\
\hline DNMT1 & NM_001379.2 & chr19:10273372 & c. $931 \mathrm{~A}>\mathrm{G}$ & I311V & rs2228612 & 0.18 & Polymorphism \\
\hline$P R X$ & NM_181882.2 & chr19:40900865 & c. $3394 \mathrm{G}>\mathrm{A}$ & G1132R & rs 268674 & 0.96 & Polymorphism \\
\hline$P R X$ & - & chr19:40902710 & c. $1549 \mathrm{C}>\mathrm{T}$ & $\mathrm{L} 517 \mathrm{~F}$ & - & - & Non-cosegregation \\
\hline$D M P K$ & NM_001081563.1 & chr19:46275976 & c. $1297 \mathrm{C}>\mathrm{G}$ & L433V & rs527221 & 0.12 & Polymorphism \\
\hline$A T P 7 A$ & NM_000052.5 & chrX:77298857 & c. $4048 \mathrm{G}>\mathrm{A}$ & E1350K & rs4826245 & 1.00 & Polymorphism \\
\hline
\end{tabular}

${ }^{\mathrm{a}}$ GenBank registration number of reference sequence. ${ }^{\mathrm{b}} \mathrm{cDNA}$ numbering was achieved with +1 , corresponding to the A of the ATG initiation

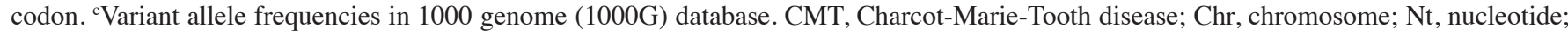
AA, amino acid.

All variants occurring in CMT relevant genes $(\sim 60)$ were initially selected. The functionally significant variants (missense, nonsense, exonic indel (insertion and deletion) and splicing site variants) were selected while the remaining variants were filtered out. Sequencing variants were confirmed by the Sanger sequencing method using an automatic genetic analyzer ABI3130XL (Applied Biosystems, Foster City, CA, USA). A mutation was considered to be an underlying cause when a candidate mutation was only located in the affected member within the family, but was not located in >200 control samples. The mutation nomenclature recommendations of the Human Genome Variation Society (http://www.hgvs.org/mutnomen/) were used to describe the variants.
In silico analysis. The conservation pattern for the protein amino acid sequence was performed using MEGA5 software, version 5.05 (http://www.megasoftware.net/) (18). Prediction of protein function affection due to amino acid substitution was performed using the online tools SIFT (http://sift.jcvi. org/), PolyPhen2 (http://genetics.bwh.harvard.edu/pph2/) and MuPro (http://mupro.proteomics.ics.uci.edu/).

\section{Results}

Identification of heterozygous missense mutation in SPTLC1. The summary of whole exome sequencing data is provided in Table I. The sequencing yield was $~ 6.14$ Gbp. Mappable 


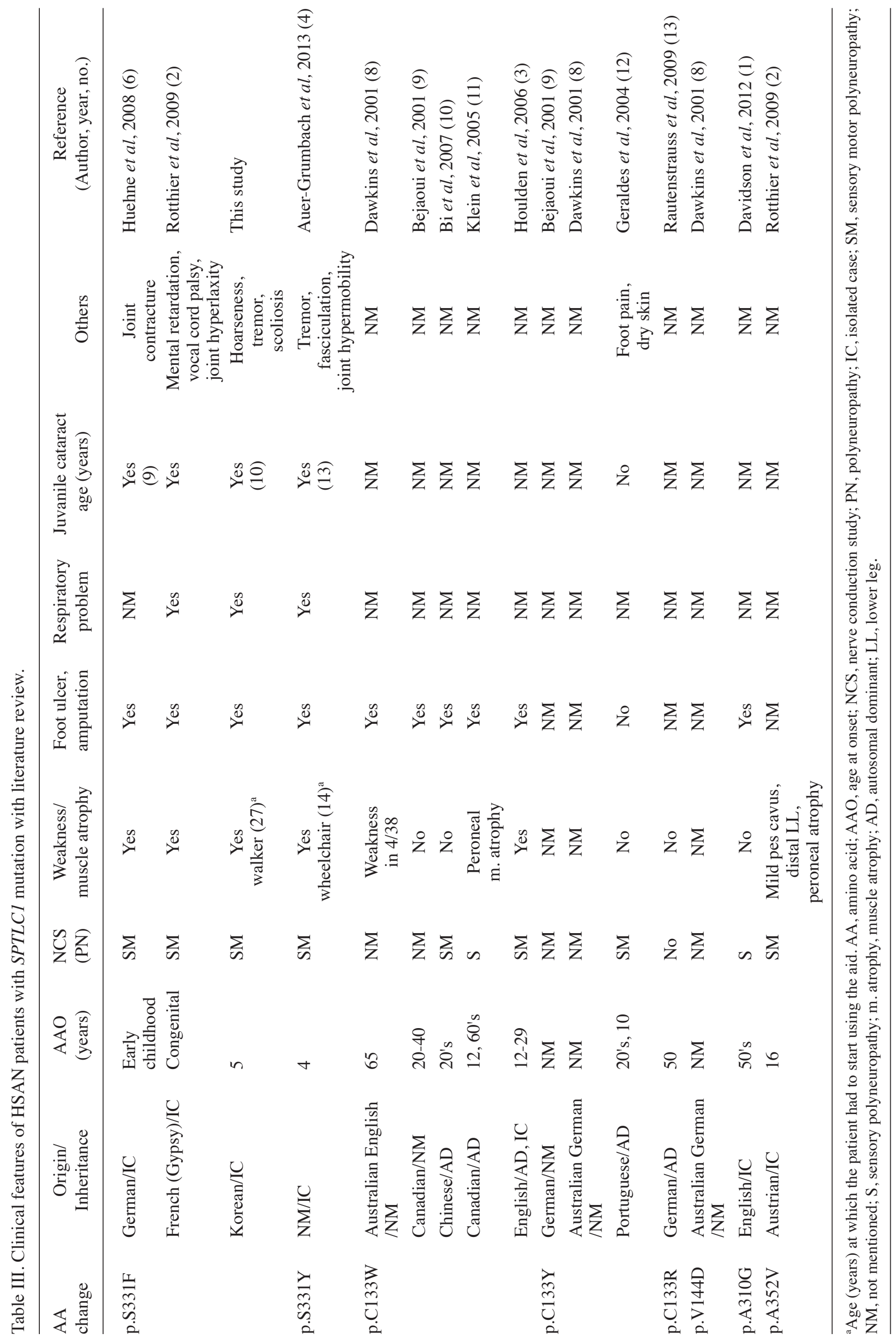


reads and target coverage ( $>10 x$ reads) were 93.1 and $94.3 \%$, respectively. Total observed numbers of SNP and indels were 57,705 and 10,450, respectively. Of these, coding SNPs and indels were 19,757 and 588, respectively.

In $\sim 60$ CMT relevant genes, 32 functionally significant variants were identified (Table II). Of these, a heterozygous missense mutation c.992C $>$ T (p.S331F) was located in the SPTLC1 gene, which was previously reported to cause HSAN I (2), and was also reported in the Inherited Peripheral Neuropathies Mutation Database (http://www.molgen.ua.ac. be/CMTMutations/). Capillary sequencing for this variant demonstrated concordant results (Fig. 1A and B). This mutation was located in neither the parents nor the sister. This result suggests that the SPTLC1 mutation in the patient occurred by de novo mutation. Notably, this case and the case reported by Rotthier et al (2) are de novo. None of the 300 controls exhibited this mutation. The SPTLC1 c.992C $>$ T (p.S331F) variant is thus determined to be the causative mutation for II-2. The mutation lies in the aspartate aminotransferase superfamily (fold type I) domain of pyridoxal phosphate-dependent enzymes.

Appreciable in silico results were attained by using three tools: SIFT yielding a deleterious score of 0.02 (damaging score: $\leq 0.05$ ), a PolyPhen score of 2.317 (damaging score: $>1.0$ ), and a MUpro protein instability score of -0.1034 (negative value: decreased stability). Analysis of multiple sequences for the conservation pattern of the STPLC1 protein demonstrated marked conservation throughout different species (Fig. 1C).

Except for SPTLC1 c.992C $>$ T, other variants in CMT relevant genes were not considered to be causative, as they were polymorphic (observed in the controls) or not cosegregated with the affected member within the pedigree. Despite the presence of several rare heterozygous variants in recessive genes, the possibility of an underlying cause was ruled out due the occurrence of the same mutation in either parent.

Clinical manifestations and electrophysiological features. The male proband (Fig. 1A, II-2) was the second child of non-consanguineous, healthy parents. Birth weight was $3.2 \mathrm{~kg}$ and motor milestones during the first year were normal. The patient noticed frequent falls, lower leg weakness and a hand tremor at age five. At seven years of age, the patient used an ankle-foot device due to a progressively impaired gait, and the diagnosis of hereditary motor and sensory neuropathy was made. Three years later, the patient had a cataract operation. Following adolescence, the voice of the patient became hoarse. Disease progression was rapid and the patient exhibited with respiratory problems and walker dependency at the age of 27 years. A neurological examination at 28 years of age revealed muscle weakness and atrophy of the proximal limb and trunk muscles (body mass index, $12.2 \mathrm{~kg} / \mathrm{m}^{2}$ ), and hypotonia with prominent weakness in the distal muscles of the upper and lower limbs (FDS 6, walking with a walker). The vibration and position senses were markedly more preserved compared with the pain and temperature senses. Marked reductions in pain and temperature sensation were noted. Bilateral hand tremors and severe scoliosis were observed. Deep tendon reflexes were absent in all extremities, but pathological reflexes were not identified.
NCS was performed at the ages of 12, 26 and 28 years. SNAPs of the median, ulnar and sural nerves were not evoked, and CMAPs of peroneal and tibial nerves were also absent. The median nerves revealed low CMAPs (range, 0.1-0.2 mV) and slow MNCVs (range, 12.2-25.9 m/s). CMAPs of the ulnar nerves were low and the range was similar to that of the median nerves (CMAP range, 0.1-0.4 mV; MNCV range, 17.5$27.0 \mathrm{~m} / \mathrm{s}$ ). However, when NCS was performed on the other family members (I-1, I-2, II-1), the results were completely normal.

\section{Discussion}

The present study reports on the early-onset of a severe phenotype of HSAN I with a missense mutation of c.992C $>$ T (p.S331F) in SPTLCl. HSAN I is characterized by autosomal dominant inheritance, juvenile or adulthood disease onset, and marked distal pain and temperature sensory impairment leading to distal ulceration and mutilating arthropathy with relative preservation of motor and autonomic functions (19). Other subtypes of HSAN (HSAN types II-V) have autosomal recessive inheritance with an earlier onset of the disease and variable motor involvement. Therefore, the phenotype of this patient was different to the usual pattern of HSAN I, with the features of: i) A sporadic case rather than autosomal dominant inheritance; ii) earlier age of onset; iii) severe proximal and distal motor involvement with wasting; and iv) additional features including cataracts at a young age, hoarseness of the voice, tremor, scoliosis and respiratory insufficiency.

To date, numerous SPTLC1 mutations have been identified at restricted amino acid positions (p.C133W,p.C133Y,p.C133R, p.V144D, p.S331F, p.S331Y, p.A310G, and p.A352V) (2-9). There have only been three previously reported cases with a mutation at the Ser331 position $(2,4-6)$. Common findings of these three cases are a de novo mutation, symptom onset prior to 10 years of age, muscle hypotrophy due to prominent motor involvement in the polyneuropathy, foot ulcers, amputations and cataracts at a young age. Two of the cases also exhibited joint hypermobility, vocal cord paralysis and respiratory difficulties $(2,4-6)$. Following a review of the literature with regard to the SPTLC1 mutation, the present case and the aforementioned three cases carrying the Ser331 mutation resemble each other in terms of the inheritance pattern and the atypical clinical features (Table III).

It is hypothesized that reduced SPT activity with resultant accumulation of neurotoxic deoxysphingoid bases (DSB) may be the underlying pathomechanism of HSAN I with SPTLCI mutations. Rotthier et al (5) demonstrated that mutant proteins (C133W, S331F and A352V) are enzymatically defective; however, failed to demonstrate a positive correlation between DSB levels and phenotypic severity. These results imply that there may be an additional factor determining the severity of the disease, including genetic modifiers or other interacting proteins.

This is the first report of a Korean HSAN I patient with the p.S331F mutation in SPTLC1. The patient exhibited early onset and severe clinical manifestations, which are uncommon features of HSAN I but are an almost identical to those in patients with an Ser331 mutation. Therefore, HSAN I with a p.S331 mutation in SPTLC1 may exhibit an early onset, severe 
sensory motor deficits and various other features, including cataracts, vocal cord palsy and respiratory problems.

\section{Acknowledgements}

This study was supported by a grant from the Korean Health Technology R\&D Project, Ministry of Health and Welfare, Republic of Korea (grant no. A120182).

\section{References}

1. Davidson G, Murphy S, Polke J, et al: Frequency of mutations in the genes associated with hereditary sensory and autonomic neuropathy in a UK cohort. J Neurol 259: 1673-1685, 2012.

2. Rotthier A, Baets J, De Vriendt E, et al: Genes for hereditary sensory and autonomic neuropathies: a genotype-phenotype correlation. Brain 132: 2699-2711, 2009.

3. Houlden H, King R, Blake J, et al: Clinical, pathological and genetic characterization of hereditary sensory and autonomic neuropathy type 1 (HSAN I). Brain 129: 411-425, 2006.

4. Auer-Grumbach M, Bode H, Pieber TR, et al: Mutations at Ser331 in the HSN type I gene SPTLC1 are associated with a distinct syndromic phenotype. Eur J Med Genet 56: 266-269, 2013.

5. Rotthier A, Penno A, Rautenstrauss B, et al: Characterization of two mutations in the SPTLC1 subunit of serine palmitoyltransferase associated with hereditary sensory and autonomic neuropathy type I. Hum Mutat 32: E2211-2225, 2011.

6. Huehne K, Zweier C, Raab K, et al: Novel missense, insertion and deletion mutations in the neurotrophic tyrosine kinase receptor type 1 gene (NTRK1) associated with congenital insensitivity to pain with anhidrosis. Neuromuscul Disord 18: 159-166, 2008.

7. Verhoeven K, Coen K, De Vriendt E, et al: SPTLC1 mutation in twin sisters with hereditary sensory neuropathy type I. Neurology 62: 1001-1002, 2004

8. Dawkins JL, Hulme DJ, Brahmbhatt SB, Auer-Grumbach M and Nicholson GA: Mutations in SPTLC1, encoding serine palmitoyltransferase, long chain base subunit-1, cause hereditary sensory neuropathy type I. Nat Genet 27: 309-312, 2001.
9. Bejaoui K, Wu C, Scheffler MD, et al: SPTLC1 is mutated in hereditary sensory neuropathy, type 1 . Nat Genet 27: 261-262, 2001.

10. Bi H, Gao Y, Yao S, Dong M, Headley AP and Yuan Y: Hereditary sensory and autonomic neuropathy type I in a Chinese family: British C133W mutation exists in the Chinese. Neuropathology 27: 429-433, 2007.

11. Klein CJ, Wu Y, Kruckeberg KE, et al: SPTLC1 and RAB7 mutation analysis in dominantly inherited and idiopathic sensory neuropathies. J Neurol Neurosurg Psychiatry 76: 1022-1024, 2005.

12. Geraldes R, de Carvalho M, Santos-Bento M and Nicholson G: Hereditary sensory neuropathy type 1 in a Portuguese family-electrodiagnostic and autonomic nervous system studies. J Neurol Sci 227: 35-38, 2004.

13. Rautenstrauss B, Neitzel B, Muench C, Haas J, Holinski-Feder E and Abicht A: Late onset hereditary sensory neuropathy type 1 (HSN1) caused by a novel p.C133R missense mutation in SPTLC1. J Peripher Nerv Syst 14: 124-125, 2009.

14. Paternostro-Sluga T, Grim-Stieger M, Posch M, et al: Reliability and validity of the Medical Research Council (MRC) scale and a modified scale for testing muscle strength in patients with radial palsy. J Rehabil Med 40: 665-671, 2008.

15. Birouk N, LeGuern E, Maisonobe T, et al: X-linked CharcotMarie-Tooth disease with connexin 32 mutations: clinical and electrophysiologic study. Neurology 50: 1074-1082, 1998.

16. Choi BO, Kim J, Lee KL, Yu JS, Hwang JH and Chung KW: Rapid diagnosis of CMT1A duplications and HNPP deletions by multiplex microsatellite PCR. Mol Cells 23: 39-48, 2007.

17. Lee SS, Lee HJ, Park JM, et al: Proximal dominant hereditary motor and sensory neuropathy with proximal dominance association with mutation in the TRK-fused gene. JAMA Neurol 70: 607-615, 2013.

18. Tamura K, Peterson D, Peterson N, Stecher G, Nei M and Kumar S: MEGA5: molecular evolutionary genetics analysis using maximum likelihood, evolutionary distance, and maximum parsimony methods. Mol Biol Evol 28: 2731-2739, 2011.

19. Auer-Grumbach M, De Jonghe P, Verhoeven K, et al: Autosomal dominant inherited neuropathies with prominent sensory loss and mutilations: a review. Arch Neurol 60: 329-334, 2003. 